\title{
Technological and methodological challenges of using classroom video to analyse physics teachers' explanations
}

\author{
David Geelan \\ School of Education \\ University of Queensland \\ Brisbane, QLD Australia
}

\begin{abstract}
This paper reports a project conducted in twelve Grade 11 Physics classrooms in Edmonton, Canada, that used close analysis of video recordings of teacher explanations to explore the ways in which physics teachers explain concepts. The focus here is on discussion of the research methods and associated technological and methodological issues rather than on the specific issue of teacher explanations. Details of particular approaches to video analysis, including the qualitative video analysis software packages vPrism and Transana are given, and the costs and advantages of using video data discussed.
\end{abstract}

Keywords: video, classroom, explanation, physics, qualitative, methodology

\section{Introduction}

How do good, successful physics teachers explain the core ideas of physics to their students? Do they do it through lecturing, through leading discussion, through almost incidental comments when discussing the results of laboratory sessions? Do they discuss the nature of science explicitly, or is it implicit in the way they describe physics concepts? What distinguishes good, clear explanations that lead to deeper understanding on the part of students from explanations that lead to alienation or incomprehension?

Further, what methods are best adapted to exploring these questions? Classroom explanations are given in the course of teaching, in classrooms. Explanations are given to someone, in a specific context and for a specific purpose. Video recordings of classroom teaching have potential to allow explanations to be analysed in detail for multiple dimensions of explanatory quality and teacher expertise.

An earlier phase of this study conducted in Perth, Western Australia (Geelan, Wildy, Louden \& Wallace, 2003; Geelan, 2003) drew my attention to issues around teacher explanations. Each of the four teachers whose teaching was videotaped and analyzed in that study had a very different personal style, and was teaching in a very different context, so their 
practices were very different. Yet each of those teachers had a history of excellent results (with very high numbers of students achieving passing grades and also high proportions of each class achieving at the highest levels) on external final examinations, and was judged by teacher peers and students to be teaching and explaining ideas in excellent ways.

In the present study, teachers in six high schools in an urban Canadian context were invited to participate in the research project. The schools were chosen on the basis of their results in the external Diploma Examinations in physics in the preceding five years. Each of the six schools had achieved very good results, in the top band for the province. The study was intended to capture and analyze the teaching practices of excellent, highly successful physics teachers.

The goal of the research was both to try to understand - in rich, complex, contextsensitive ways - the key features of good physics teacher explanations. A secondary goal of the project was to be able to use video vignettes in combination with the understandings developed in the course of the research to show these features of excellent explanations to students in teacher preparation programs. In other words, in this study annotated video was both one of the methods by which the research was conducted and one of the methods by which the findings were to be communicated. Reducing the richness of real explanations and their associated codes to text or lecture was regarded as an abstraction that robbed them of much of their power, and using the video material directly as a more authentic means of conveying ideas about the quality of explanations.

\section{Explanatory Frameworks}

Science teachers' explanatory frameworks - the way they use analogy, metaphor, examples, axioms and concepts, and the way these elements are tied together into a coherent whole are an increasing focus of interest in science education research (Treagust \& Harrison, 1999, 2000). This work is related to more than one of the dimensions of teacher expertise identified by Hattie (with a number of collaborators) (Bond, Smith, Baker \& Hattie, 2000; Hattie, Clinton, Thompson, \& Schmidt-Davis, 1995, 1996).

The nexus of the discussions in science education about explanatory frameworks with those in education more generally about teacher expertise, combined with the new analytical tools made available by video analysis, provide an area for research that is potentially highly fruitful in delivering rich understanding of what constitutes excellence in physics instruction. Such understandings in turn have the potential to be fruitfully introduced into the pedagogy of science teacher education, including the use of actual classroom video in prompting 
prospective teachers' critical reflection on issues related to expertise in science teaching.

Scientific explanations are law-like, logical and highly generalized. Science teaching explanations, however, can be placed on a continuum between science explanations and 'everyday' explanations. Science teaching explanations can draw on analogy, anthropomorphism and teleology. Their key goals are to connect with students' prior understandings and help students develop 'fluid mental models' of scientific concepts (Treagust \& Harrison, 2000).

\section{Research Methods}

The research approach uses multiple methods of both collecting and interpreting the research data (Geelan \& Hopkins, 2002). Schools within the Edmonton Public School Board were chosen on the basis of their highly successful physics results in the external diploma examinations, published on the Alberta Learning web site. The principals of selected schools were contacted, and permission sought to contact the Head of Department of science and the physics teachers in the school, in order to seek their participation in the study. Where teachers agreed, between 3 and 5 hours of videotape were recorded in their physics classrooms. Each teacher was recorded with one class group. Teachers, parents and students were also surveyed on issues related to teacher explanations and expertise, teachers interviewed, and focus groups of 5-6 students participated in discussion of the issues around teacher explanations. These focus group discussions were audiotaped and transcribed for analysis.

A grounded theory approach (Charmaz, 2000, 2001) was adopted when coding the video lessons (interview video and audio was transcribed separately and are not discussed in this paper). That is, the video was transcribed and repeatedly watched, and themes that occurred in multiple lessons and multiple classrooms listed. A hierarchy of emergent themes and issues was developed, and then each video segment was coded, as described below. The process of constructing categories and coding video was iterative, and was continued until the coding scheme was sufficiently comprehensive to capture all of the key features of the explanations offered in the lessons.

It could be argued that the methodological approach adopted was not 'pure' grounded theory, in the sense that the watching of the video was informed by the researchers' prior reading of the Treagust and Harrison $(1999,2000)$ and Ruben $(1990,1993)$ discussions of explanation and by Hattie and his coauthors' (1995, 1996, 2000) discussions of teacher expertise. Since the purpose of the study was to focus on explanation and expertise, and since 
some codes arose that were not based in those frameworks, it is considered that this is not a serious methodological flaw in the study.

\section{Ethics and Access}

Research ethics clearance for the study was obtained from the Research Ethics Board at the University of Alberta. Research in schools is always more difficult from the perspective of gaining ethics clearance because it involves minors, who cannot give informed consent to participate in research. Consent must be obtained from their parents or guardians. In this study parents were asked to read an information letter and sign a consent form. Students were asked to sign the same form as their parents, partly in order to ensure that they were willing to participate and partly as a courtesy to them so that they felt they had been consulted.

The use of video added an extra layer of difficulty because one of the usual standards used in research ethics is the non-identifiability of participants. This is easy to achieve using codes in quantitative studies and pseudonyms in qualitatative research, but video footage of a student is difficult to render non-identifiable. The approach taken was that the video would not be used with or shown to groups who might be able to make the connection between a student's image and his or her name and identity.

Yet another layer was added because of the dual purpose use of the video - as well as being used in the research project to be analysed to better understand teachers' explanations, permission was sought to use the video in teacher education courses to present the results of the analysis to beginning teachers. The consent forms used for all participants included a check box that allowed them to opt in or out of this second use of the video. Fortunately for the research team all participants checked this box.

Since the focus of the recording was on the teachers, students' appearances in the video recordings were only incidental - as they entered or left the room, or went to the teacher's desk to ask for help or to the board to solve a problem. Students for whom consent had not been gained were placed at the sides of the classroom to reduce the probability that they would appear in the video, and the camera turned off when they were moving around the classroom, or if they were inadvertently filmed the tape was edited to avoid their image appearing. Sometimes, in order to retain the continuity of the audio, this meant grafting another image or piece of video into the video track of the file while keeping the audio running. 
One ethical issue relates to the impact of the video recording on the students' learning in class. In the earlier Perth study some teachers were very nervous in the presence of the camera, and this may have impacted on their teaching, although it is also possible that it led them to prepare more thoroughly for their classes and bring in more interesting demonstrations to show the students. In general the Canadian teachers very quickly become comfortable with, and then oblivious to, the presence of the cameras, and simply taught as they would normally have done (this was confirmed in interviews with the students). Another potential impact was moving students for whom consent to appear in the videos had not been gained to the sides of the classroom, but in most of these classrooms students did not regularly sit in particular seats, so moving them was not perceived to have a major impact on their learning. A further ethical issue was that it was impossible in this study for students to entirely opt out of the research project - even if they did not consent to participate in the project they remained in a class in which the project was taking place. Given the very limited effects of the recording on the activities of the classroom this was considered to be an ethically acceptable situation.

One unanticipated problem that consumed a large amount of editing time was that a graduate student who was an experienced teacher was used to record some of the lessons. He obtained the consent forms from those students and parents who completed them, but then assumed that this meant he could record all members of the class without keeping track of which students had not given consent. Since there was no method of linking students' images with their names on the consent forms, it was impossible to go back and edit out only those students who had not given consent, so all students' images had to be actively edited out or obscured in all video recorded by that student. Careful, clear and explicit briefing of all research team members can save dozens of hours of work.

The novelty of using video recording as part of a social science research project, combined with the issues of identifiability raised by the medium, meant that it was much more difficult to have the study given clearance by the University of Alberta Faculty of Education's Research Ethics Board (REB - the equivalent of US universities' Institutional Review Boards (IRBs)). Studies using video are consistently difficult to deal with and required significant discussion. The REB was usually able to put in place additional provisions and safeguards that made it possible for the studies to go ahead, but it is worth noting that obtaining ethical clearance for video studies may take extra time and work.

Similarly, obtaining 'gatekeeper' permission from the Edmonton Public School Board's research officer also took extra time and quite a few phone calls back and forth. Unexpectedly, 
one of the issues that arose with the school board was the concern that the research team might be planning to make money from selling the classroom video we recorded. It was possible to reassure them on that point.

\section{Technological Solutions}

The collected video footage was converted to digital form, transcribed and analyzed for explanatory frameworks and pedagogical strategies.

Digital video is very demanding of permanent (e.g. video tape or hard drive) memory capacity - an hour of uncompressed video is equivalent to approximately 15 GB of data. Since at the time most of the computers on which we were working had 120 GB hard drives the 60 hours of video in the study needed to be compressed if it was to be manageable. The original MiniDV tapes of all lessons were kept as a backup, but all video was uploaded to the computer and then Apple's Quicktime Pro was used to compress it so that each hour of video was in the vicinity of $500 \mathrm{MB}$. This made it possible to fit each hour onto a single CD (the dominant backup storage medium before DVD burners became common) and made the whole study more manageable at about $30 \mathrm{~GB}$ of video data. If this study were being conducted today, with much larger hard drives and with dual layer DVD burners common and BluRay DVD just around the corner for consumer use, quite a bit of time spent juggling storage space would be avoided.

Once the video had been digitised and compressed it was imported into a qualitative video analysis software package and transcribed.

The first phases of the study were conducted using LessonLab's 'vPrism', a software package that allows parallel coding and analysis of the video and the transcript. vPrism was available for Windows and for MacOS 9. This meant it could still be used under MacOS X using the 'Classic' environment, but only on PowerPC-based Macs. Even at that stage it had been essentially abandoned by LessonLab in terms of updates and support. When Apple moved to Intel-based computers the Classic environment was no longer accessible, which meant that vPrism was no longer a usable option. vPrism had been a very powerful program, with more capabilities than were really needed for this project, but it was also difficult to use, with a steep learning curve, and had some quirks that would tend to lock the computer up on a regular basis. It also required all video data to be cut into pieces less than 58 minutes long, which further fragmented the data set.

Once vPrism become inaccessible for use, the research team chose to shift the project to Transana, an open-source software package available for both Mac and Windows from 
http://www.transana.org and developed at the University of Wisconsin-Madison Center for Educational Research. Unlike vPrism, Transana is designed for educational research. It is simpler to learn and operate, and although it has fewer 'bells and whistles' than vPrism its flexibility and excellent levels of support meant the project proceeded much more easily once Transana was used to transcribe, code and analyse the data.

\section{Coding Scheme}

Important features of the analysis software included the ability to code for both single instants and for extended sections of video from a few seconds to a few minutes, as well as the ability for codes to overlap. For example, a particular section of video might exemplify both open-ended questioning and the use of an analogy, and both things needed to be coded for analysis.

Specific features coded included:

- the use of analogies and metaphors, with a separate code where teachers paid explicit attention to the ways in which the analogical concept was unlike the target concept (i.e. the places where the analogy breaks down),

- instances where teachers worked through calculations on the board,

- use of diagrams and of electronic animations or simulations,

- use of apparatus and demonstrations in explaining,

- use of anthropomorphic and teleological language,

- teachers' use of hand gestures or body movements to illustrate their explanations,

- appeals to earlier learning in the course,

- drawing on knowledge from other courses the students are taking (usually, but not always, mathematics),

- explicit allusions to assessment such as 'you'll need to know this for the test' or 'this is how I would do this problem in an exam',

- use of questions including open- and closed-ended questioning,

- jokes and humor, including pop-culture allusions.

Time coding could potentially have been used as a means of adding a quantitative dimension to the video analysis - measurement of the proportion of time spent doing calculations on the board, versus helping students with calculations in their seats, for example. This was not done, simply because the video recording of the lessons occurred on a somewhat opportunistic schedule, when it was possible for the researchers to get to the school, and since the focus of the study was on teacher explanations the camera was often turned off 
during student experiments or 'seatwork'. This meant that there was no sensible 'timeline' for the study in each classroom as a whole to which particular activities could be compared to analyse the proportion of time spent on them. This meant that the study was entirely a qualitative analysis of the features of teacher explanations as they were observed in the recorded classes.

\section{Why Video?}

In comparison with other possible methods for accessing teacher explanations, such as classroom observation using a coding scheme or some form of textual analysis applied to the transcripts of teachers' explanations, video had additional costs. Audio recording lessons and transcribing them would have taken as much transcription time but been less expensive in terms of media such as hard drive space, consumables like MiniDV tapes and time to compress the video files. Observation would have required less time spent on tasks other than direct observation of teaching, and could therefore have increased the number of hours of teaching that could be analysed.

So, given these additional costs, why use video rather than other available approaches? Video offered several advantages for this project.

Access to gesture and demonstrations - explanations were sometimes purely verbal, and for such explanations an analysis of the textual transcript would likely be sufficient. Many times, however, the teacher either used hand gestures to illustrate features of the phenomenon being described, drew diagrams or solved equations on the board, showed a demonstration using a piece of apparatus or used a computer-based animation or simulation as part of an explanation. The explanation, then, did not simply consist of the words, with these other visual media as mere illustration. Rather, the explanation was made up of both the visual and audible dimensions, and video as a medium was capable of capturing this feature of science teaching explanations in ways impossible for alternative methods.

Multiple passes and different speeds - when classroom observation is conducted in 'real time' the researcher does not have the option of pausing the teacher's explanation to take a break, or of rewinding a section to check whether a particular phrase was heard correctly. Both audio and video recordings do offer this 'time shifting' capability, but with video there is the additional benefit of being able to use lip-reading and gesture to interpret unclear phrases. Video can also be slowed down or sped up in order to pay attention to particular features of the phenomenon of interest. A particular segment can be played through in its entirety and coded for a particular dimension of interest, then run through again and coded 
for a different dimension. This reduces the cognitive complexity of coding, since in a real time approach the coder has to attend to all codes simultaneously, and improves reliability.

Participant corroboration - the use of video makes it possible to take a particular lesson or instance back to the teacher who is depicted in it and ask for clarification on any issue. For example, a researcher can ask about the teaching intentions the teacher had for that session, and the ways in which they were embodied in the teaching or muffled through external constraints and issues or through the students' behaviour. Teachers can be interviewed about their practices in the light of very concrete examples of that practice. (This affordance of video was not used in this study because it was considered to be too much of a time commitment to ask of teachers.) At a higher level, interpretations of particular pieces of video can be checked with participants for the fairness (or otherwise) with which they feel their practices and intentions are represented.

Multiple overlaid codings - a related issue is the possibility of coding a particular lesson segment as an instance of two or more categories - perhaps as an open-ended question and the process of testing students' developing mental models of a phenomenon. While it is theoretically also possible to perform this kind of coding in a real time classroom observation it seems improbable that it would be possible to do so consistently for long sessions of observation, and there is no mechanism by which codings can be checked later for consistency.

Persistent data - the dynamic and iterative nature of the grounded theory-based approach used in this study meant that categories for coding emerged both during the initial phases of the study and during the coding process itself. Rather than all the categories being specified ahead of time and embodied in a coding protocol, new categories of interest appeared as the video was watched again and again and coded. The persistence of the video data meant that it was possible to go back through already-coded segments of video and code them for the new emergent categories. Even more than this, if new areas of research interest or new conceptual frameworks for understanding teachers' classroom explanations appear in the science education literature in future it would still be possible to recode and reanalyse the persistent video data to seek supportive or disconfirming evidence for these new schemes. This is a feature of video data that is to some extent shared by textual analysis of transcripts or audio data, but when combined with the additional visual cues discussed above makes the use of video a particularly valuable method.

These are the research advantages of using video, however there was an additional benefit foreseen in the ability to communicate the findings of the study. 


\section{Classroom Video In The Classroom}

Explanations in physics education are often made up of much more than the words involved. Recognising this meant that communicating the findings of this research project, both at academic conferences and to beginning teachers in teacher education courses, was better served by the thoughtful use of classroom video than by simple verbal explanations of the frameworks and features identified in the study. Rather than telling colleagues and students about the features of a teacher's use of a spoon, his drinking water bottle and a wineglass to demonstrate features of waves in a closed column of various lengths to students, it was more powerful to show a short video vignette. This use of video brings much more of the context of a particular school classroom to the teacher education classroom - which is crucial because an explanation is given to someone in particular. It can even be argued that an explanation cannot really exist without the presence of a person whose understanding it is intended to enhance (this is, of course, a question philosophers have discussed at length, e.g. Ruben, 1990, 1993).

Both vPrism and Transana allow collections of clips that are identified with the same code to be pulled together and shown as a single longer video segment. This is important because it allows multiple examples of a particular category or theme to be shown to students. If a single exemplar of a particular concept is shown it is easy for novice observers such as beginning teachers to focus on extraneous elements of that particular instance, such as the teacher's classroom manner (or even his tie!) and to miss the key concept (e.g. the use of a diagram to elicit students' misconceptions in relation to a particular process) that is being presented. The use of a number of different, linked clips showing different instances of the same concept can allow students to come to identify what is common to the clips, and therefore to be able to identify that feature of explanations in others' and their own teaching practice. The ability to name and identify these quite sophisticated elements of physics teaching explanations is an important part of the process of developing their pedagogical content knowledge (Shulman, 1986) in physics teaching, and I would argue that this use of classroom video has considerable potential impact in science teacher education.

\section{Conclusion}

The use of classroom videotapes, combined with a qualitative analysis software package such as vPrism or Transana, has the potential to allow researchers to analyze in detail the fastmoving practices of classroom teachers, the professional judgments that inform those 
practices from moment to moment (Griffiths and Tann (1992) refer to such judgments as 'reflection in action'), and the explanatory frameworks that help teachers to increase students' understanding of difficult concepts. By drawing attention to, and providing concrete, contextsensitive, authentic examples of such explanatory frameworks and their use in the classroom, such videotapes and the associated understandings also have the potential to enrich teacher education, through giving beginning teachers access to the strategies and approaches of experienced teachers.

\section{References}

Bond L, Smith T, Baker WK and Hattie JA (2000) The certification system of the National Board for Professional Teaching Standards: A construct and consequential validity study. Greensboro, NC: Centre for Educational Research and Evaluation, The University of North Carolina at Greensboro.

Charmaz K (2001) Qualitative interviewing and grounded theory analysis. In Gubrium J and Holstein J (Eds) Handbook of interview research: Context and method, pp. 675-694.

Charmaz K (2000) Grounded theory: Objectivist and constructivist methods. In Denzin N and Lincoln Y (Eds) Handbook of qualitative research (2nd ed), pp. 509-535.

Geelan DR, Wildy H, Louden W and Wallace J (2003) Teaching for understanding and/or teaching for the exam in high school physics. International Journal of Science Education, 26(4): 447-462.

Geelan DR (2003) Teacher expertise and explanatory frameworks in a successful physics classroom. Australian Science Teachers Journal , 49(3): 22-32.

Geelan DR and Hopkins SL (2002) Multimethod approaches for research in science and mathematics education. Alberta Science Education Journal, 34(2): 3-14.

Griffiths M and Tann S (1992) Using reflective practice to link personal and public theories. Journal of Education for Teaching, 18(1): 69-84.

Hattie J, Clinton J, Thompson M and Schmidt-Davis H (1995) Identifying highly accomplished teachers: A validation study. Greensboro, North Carolina: Centre for Educational Research and Evaluation, University of North Carolina - Greensboro.

Hattie JA, Clinton JC, Thompson M and Schmidt-Davis H (1996) Identifying expert teachers. Paper presented at the North Carolina Association for Research in Education, Chapel Hill, NC.

Ruben D-H (1993) Explanation. Oxford: Oxford University Press.

Ruben D-H (1990) Explaining Explanation. London and New York: Routledge. 
Shulman LS (1986) Those who understand: Knowledge growth in teaching. Educational Researcher, 15(2): 4-14.

Treagust DF and Harrison AG (2000) In search of explanatory frameworks: an analysis of Richard Feynman's lecture 'Atoms in motion'. International Journal of Science Education, 22(11): 1157-1170.

Treagust DF and Harrison AG (1999) The genesis of effective scientific explanations for the classroom (pp. 28-43). In Loughran JJ (Ed) Researching teaching: Methodologies and practices for understanding pedagogy. London: Falmer Press. 